\title{
On numerical simulations of flow and heat transfer of the bend part of a U-duct
}

\author{
T. Salameh \& B. Sundén \\ Department of Energy Sciences, Division of Heat Transfer, \\ Lund University, Sweden
}

\begin{abstract}
Two-dimensional numerical simulations of the flow and temperature fields inside the bend (turn) part of a U duct have been performed. Both the standard and low Reynolds number k- $\varepsilon$ models were used to solve the smooth bend (turn) part and ribbed bend (turn) part, respectively. For the standard k- $\varepsilon$ model, the wall function approach was used at the near wall region where the log-law was assumed to be valid, whereas the modelling damping functions were used in the low Reynolds number model. In the case of the ribbed bend (turn) part, two approaches were used, the total approach and an approach based on periodic flow condition. The details of the duct geometry were as follows: the cross section area of the straight part is $50 \times 50 \mathrm{~mm}^{2}$, the inside length of the bend part $240 \mathrm{~mm}$, the cross section area of the rib is $5 \times 5 \mathrm{~mm}^{2}$ and the rib height-tohydraulic diameter ratio, $\mathrm{e} / \mathrm{Dh}$, is 0.1 . The results are compared with experimental data obtained for the same conditions.
\end{abstract}

Keywords: smooth and ribbed bend part, standard $k-\varepsilon$, low Reynold number $k-\varepsilon$, periodic flow condition.

\section{Introduction}

Roughness elements (ribs or turbulators) are used to enhance the heat transfer level in the cooling passage where the cooling is required. The ribs enhance the heat transfer process by restarting the boundary layer after flow reattachment between ribs as shown in Fig. 1. 


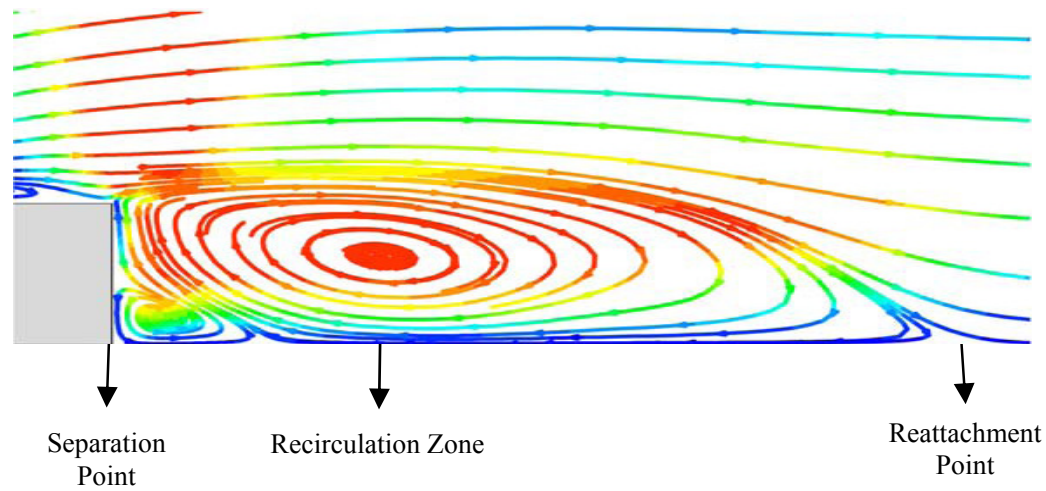

Figure 1: $\quad$ Flow regime downstream a rib.

Many numerical investigations have been performed to study both flow and heat transfer fields inside various ribbed duct shapes. Some researchers used a straight duct to investigate the effect of roughness elements (ribs) on both flow and heat transfer fields. Acharya et al. [1] used the nonlinear and standard k- $\varepsilon$ turbulence models and the nonlinear model was found to predict more realistic Reynolds stresses in the core flow region immediately above the ribs than the standard k- $\varepsilon$ model. Liou et al. [2] performed calculations with the k- $\varepsilon-A$ PDM turbulence model together with a smoothed hybrid central/skew upstream difference scheme (SCSUDS) and the PISO pressure-velocity coupling algorithm was applied to solve the accelerated, separated and recirculating flows. Prakash and Zerkle [3] predicted numerically the turbulent flow and heat transfer by using the k- $\varepsilon$ model in conjunction with wall functions. Ooi et al. [4] investigated the accuracy of modern eddy-viscosity type turbulence models in predicting turbulent flow and heat transfer in complex passages.

Other researchers used a fixed $U$ bend (turn) duct, sometimes called a $180^{\circ}$ sharp end duct to study the effect of the bend (turn) part of $U$ duct on both flow and heat transfer fields. Besserman and Tanrikut [5] evaluated two near-wall shear-stress treatments (wall functions and the two layer wall integration method) in conjunction with the k- $\varepsilon$ formulation of turbulence to assess their ability to predict high local gradients in heat transfer. Iacovides [6] applied the effective viscosity (EVM) and second-moment turbulence models in the computation of flow and heat transfer through rib-roughened passages. Sewall and Tafti [7] presented large eddy simulations of a 180 deg bend in a stationary ribbed duct. Two cases were compared: one included a rib in the bend while the other did not.

Furthermore, both rotating straight and $U$ bend (turn) ducts were used to study the effect of rotation, buoyancy and the Coriolis force in the centrifugal acceleration field. Murata and Mochizuki [8] investigated the effect of Coriolis buoyancy forces on turbulent heat transfer by rib turbulators by using large eddy simulations (LES). Murata and Mochizuki [9] simulated numerically the heat 
transfer in a rotating rib-roughened rectangular duct by using large eddy simulations with a Lagrangian dynamic subgrid-scale model.

Although a lot of numerical investigations have been performed to study the flow field and heat transfer field inside U-duct geometries, only limited details of the flow and heat transfer fields on the smooth and ribbed bend (turn) part of Uducts are available. Furthermore, most previous studies were related to the flow field and heat transfer field on the leading and trailing walls of the two-pass channels, and very limited information is available for the bend (turn) part of the U-duct. For these reasons, it is desirable to investigate more details of the flow and heat transfer fields across both the smooth and ribbed bend (turn) parts of Uducts.

\section{Computational model}

Figure 2 shows the computational domain used for studying the bend (turn) part of a $\mathrm{U}$ duct. The U-duct consists of a $1500 \mathrm{~mm}$ long straight part followed by a $240 \mathrm{~mm}$ inside length for the bend (turn) part with cross section area $50 \times 50 \mathrm{~mm}^{2}$. The ribs were placed transversely to the direction of the main flow at the outer and inner walls of the bend (turn) part of the U-duct in different arrangement. The cross section area of the rib was $5 \times 5 \mathrm{~mm}^{2}$ and the rib height-to-hydraulic diameter ratio, e/Dh, was 0.1 . The outer wall of the bend part was heated by using constant temperature boundary condition. The thermal conductivity (k) for both U-duct walls and ribs were $0.2 \mathrm{Wm}^{-1} \mathrm{~K}^{-1}$.

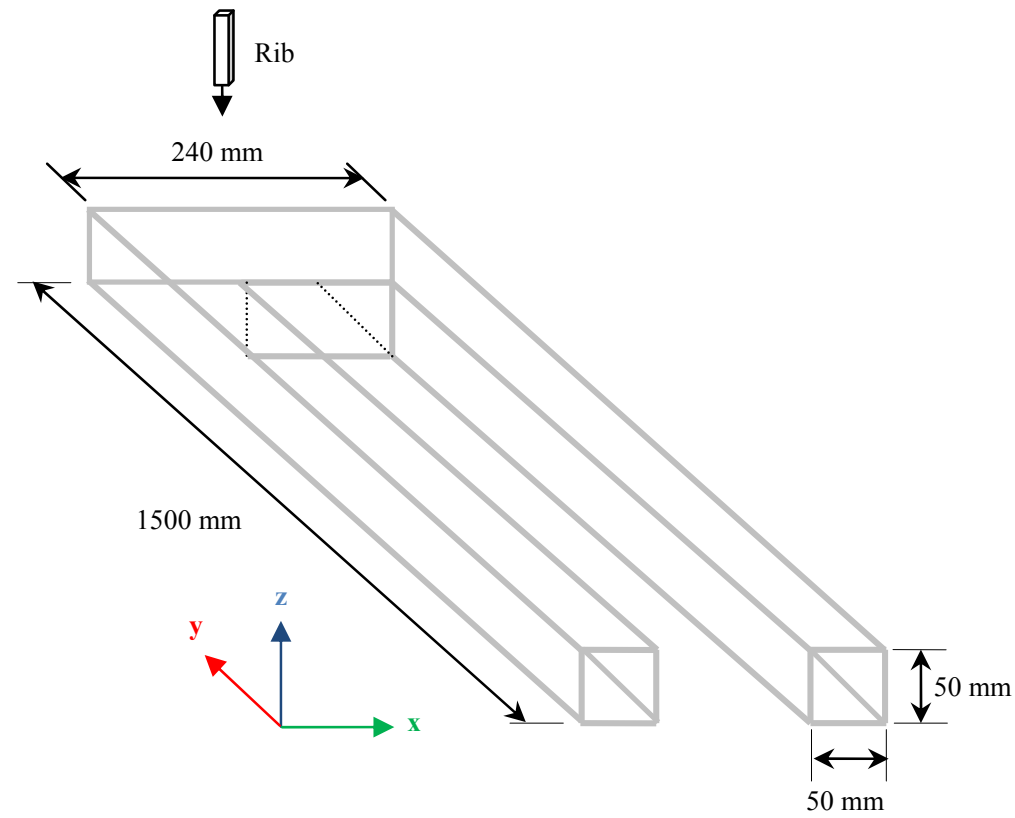

Figure 2: Schematics of the computational domain. 


\section{Governing equations}

The SIMPLEHT heat transfer computer code was used to solve the two dimensional distribution for the flow and temperature fields inside the bend (turn) part of a $U$ duct. The code uses the finite volume method to discretize the governing equations for both the flow and temperature fields with appropriate boundary conditions. The power law scheme was used to calculate the convective terms at the cell faces of control volumes whereas the SIMPLE algorithm was used to link the pressure and velocity fields (pressure-velocity coupling). Both the standard and low Reynolds number k- $\varepsilon$ models were used to solve the smooth bend (turn) part and ribbed bend (turn) part, respectively. The equations for the $\mathrm{k}-\varepsilon$ model are shown in Table 1.

Table 1: Governing equations for the k- $\varepsilon$ model.

\begin{tabular}{|c|c|c|c|}
\hline $\multicolumn{4}{|c|}{\frac{\partial}{\partial x}(\rho u \phi)+\frac{\partial}{\partial y}(\rho v \phi)}=\frac{\partial}{\partial x}\left(\Gamma_{\phi} \frac{\partial \phi}{\partial x}\right)+\frac{\partial}{\partial y}\left(\Gamma_{\phi} \frac{\partial \phi}{\partial y}\right)+S_{\phi}$ \\
\hline Equation & $\phi$ & $\Gamma_{\phi}$ & $S_{\phi}$ \\
\hline Continuity & 1 & 0 & 0 \\
\hline $\mathrm{U}$ momentum & $u$ & $\mu+\mu_{t}$ & $-\frac{\partial p}{\partial x}+\frac{\partial}{\partial x}\left(\Gamma_{\phi} \frac{\partial u}{\partial x}\right)+\frac{\partial}{\partial y}\left(\Gamma_{\phi} \frac{\partial v}{\partial x}\right)$ \\
\hline V momentum & $v$ & $\mu+\mu_{t}$ & $-\frac{\partial p}{\partial y}+\frac{\partial}{\partial x}\left(\Gamma_{\phi} \frac{\partial u}{\partial y}\right)+\frac{\partial}{\partial y}\left(\Gamma_{\phi} \frac{\partial v}{\partial y}\right)$ \\
\hline \begin{tabular}{c} 
Temperature \\
\hline $\begin{array}{c}\text { Turbulent } \\
\text { kinetic energy }\end{array}$
\end{tabular} & $k$ & $\mu+\mu_{t} / \sigma_{k}$ & 0 \\
\hline $\begin{array}{c}\text { Dissipation } \\
\text { rate }\end{array}$ & $\varepsilon$ & $\mu+\mu_{t} / \sigma_{\varepsilon}$ & $f_{1} C_{1 \varepsilon} \frac{\varepsilon}{k} P_{k}-f_{2} \rho C_{2 \rho} \frac{\varepsilon^{2}}{k}$ \\
\hline
\end{tabular}

The production term for the turbulent kinetic energy can be represented in two dimensional flow as

$$
P_{k}=\mu_{t}\left(2\left(\frac{\partial u}{\partial x}\right)^{2}+\left(\frac{\partial u}{\partial y}+\frac{\partial v}{\partial x}\right)^{2}+2\left(\frac{\partial v}{\partial y}\right)^{2}\right)
$$


The turbulent viscosity is calculated as

$$
\mu_{t}=f_{\mu} \rho C_{\mu} \frac{k^{2}}{\varepsilon}
$$

The others modelling constants are as follows

$$
\operatorname{Pr}_{t}=0.9 ; \sigma_{k}=1.00 ; \sigma_{\varepsilon}=1.30 ; C_{\mu}=0.09 ; C_{1 \varepsilon}=1.44 ; C_{2 \varepsilon}=1.92
$$

For the low-Reynolds number $\mathrm{k}-\varepsilon$ model, the damping functions $f_{1}, f_{2}$ and $f_{\mu}$ are used as shown in Table 2 .

Table 2: Damping functions.

\begin{tabular}{|c|c|}
\hline$f_{\mu}$ & $\left(1-\exp \left(-0.0165 \mathrm{Re}_{y}\right)\right)^{2}\left(1+20.5 / \mathrm{Re}_{t}\right)$ \\
\hline$f_{1}$ & $1+\left(0.05 / f_{\mu}\right)^{3}$ \\
\hline$f_{2}$ & $1-\exp \left(-\mathrm{Re}_{t}^{2}\right)$ \\
\hline
\end{tabular}

where $\operatorname{Re}_{t}=\rho k^{2} / \varepsilon \mu$ and $\operatorname{Re}_{y}=\rho \sqrt{k} y / \mu$.

For the standard k- $\varepsilon$ model these damping functions are set as unity and the wall function approach is used at the near wall region where the log-law is assumed to be valid, the following wall functions were used in the simulations for both the flow and temperature fields, respectively.

$$
\begin{aligned}
& u^{+}=\frac{1}{\kappa} \ln \left(E y^{+}\right), \quad k=\frac{u_{\tau}^{2}}{C_{\mu}^{0.5}}, \quad \varepsilon=\frac{u_{\tau}^{3}}{\kappa y} \\
& T^{+}=\operatorname{Pr}_{t}\left[u^{+}+9.24\left\{\left(\frac{\operatorname{Pr}}{\operatorname{Pr}_{t}}\right)^{0.75}-1\right\} \times\left\{1+0.28 \exp \left(-0.07 \frac{\operatorname{Pr}}{\operatorname{Pr}_{t}}\right)\right\}\right]
\end{aligned}
$$

The von Karman constant $\kappa=0.41, \mathrm{C}_{\mu}=0.09$, the wall roughness parameter $\mathrm{E}=$ 9.8. $\mathrm{y}$ is the normal distance to the solid wall and the friction velocity is $u_{\tau}=\sqrt{\tau_{w} / \rho}$.

Table 3: $\quad$ Wall shear stress.

\begin{tabular}{|c|c|}
\hline$y^{+}=\frac{\rho \Delta y_{P}}{\mu} \sqrt{\tau_{w} / \rho}$ & $\tau_{w}$ \\
\hline$y^{+}<11.63$ & $\mu u_{P} / \Delta y_{P}$ \\
\hline$y^{+}>11.63$ & $\rho u_{\tau}\left(u_{p} / u^{+}\right)$ \\
\hline
\end{tabular}


The wall functions were applied at the grid point adjacent to the solid wall and implemented as source terms in the control volume adjacent to the wall for the momentum ( $u$ velocity) equation parallel to the wall, k-equation, $\varepsilon$-equation and temperature equation. The wall shear stress was calculated based on the dimensionless normal distance to the wall $\mathrm{y}^{+}$as shown in Table 3 .

In the case of the ribbed bend (turn) part, the periodic flow approach was used by expressing the pressure in a periodic manner from cycle to cycle as

$$
p(x, y)=-\beta x+p^{*}(x, y)
$$

Here $\beta$ is a constant source term representing the non-periodic pressure gradient in the flow direction, at the inlet and outlet boundaries, the periodic condition $\phi(\mathrm{x}, \mathrm{y})=\phi\left(\mathrm{x}+\mathrm{L}_{\mathrm{cycl}}, \mathrm{y}\right)$ is imposed, where $\phi=\mathrm{u}, \mathrm{v}, \mathrm{P}^{*}, \mathrm{k}, \varepsilon$ and $\mathrm{T}$.

\section{Results and discussion}

\subsection{Low Reynolds number $k-\varepsilon$ model}

The preliminary two-dimensional distribution results for both velocity and temperature inside the smooth bend (turn) part of the U-duct are shown in Fig 3 (a), where both the velocity boundary layer and thermal boundary layer are clearly observed. The velocity of the flow has the highest value at the middle of bend (turn) part (core region of the flow) where the inertia force is dominating over the viscous force which dominates near to the inner and outer walls of the bend (turn) part of the U-duct.

For the temperature profile, the highest value exists at the outer wall of the bend (turn) part of the U-duct where the constant wall temperature boundary condition is applied. In Figs. 3 (b) and (c), the presence of the ribs along the bend (turn) part of the U-duct changes both the velocity and thermal boundary layers. The highest value of the velocity exists at the core region of the flow whereas the negative values appear downstream the rib where the recirculation zone occurs. The thickness of the thermal boundary layer was reduced compared to case (a).

Figure 3(d) shows the effect of 5 ribs along the bend (turn) part of the U-duct on both the velocity boundary layer and thermal boundary layer. The velocity increases more than for case (c) and negative velocities appear behind the ribs. The thermal boundary layer starts to expand again as can shown in temperature distribution.

\subsection{Low Reynolds number $k-\varepsilon$ model based on periodic boundary condition}

Figure 4 shows the preliminary two-dimensional distributions for both velocity and temperature inside the ribbed bend (turn) part of the U-duct based on the one cycle approach for periodic flow. Both velocity and temperature distributions agree with the total cycles as explained before. The very thin thermal boundary layer obtained at the outer wall of the U-duct where the constant wall temperature boundary condition was applied. 

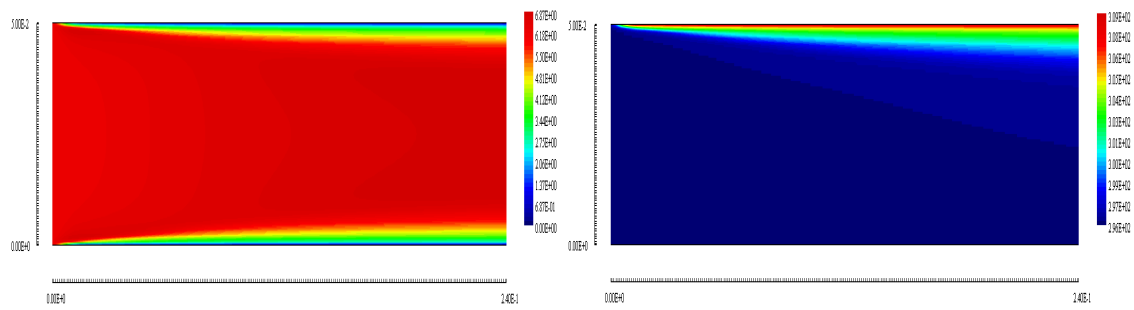

(a)
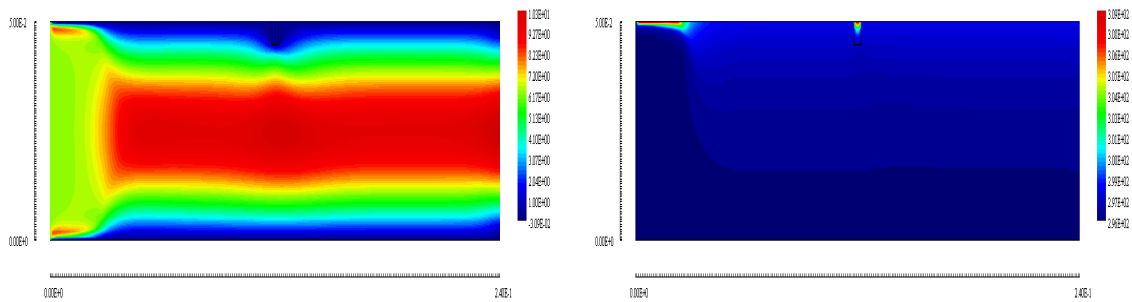

(b)
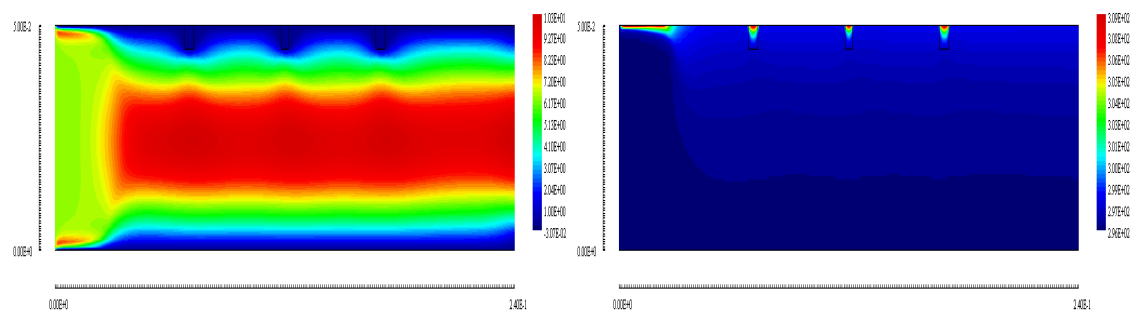

(c)
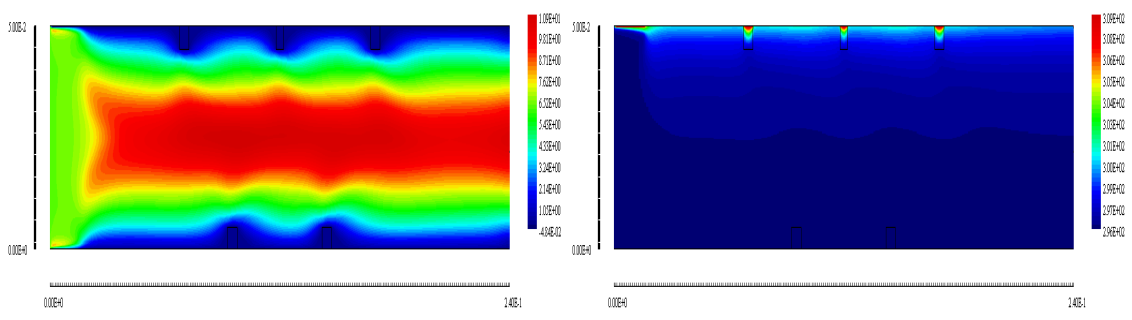

(d)

Figure 3: U-velocity and temperature distributions by the low Reynolds number k- $\varepsilon$ model. (a) Smooth (b) 1-Rib (c) 3-Ribs (d) 5-Ribs. 


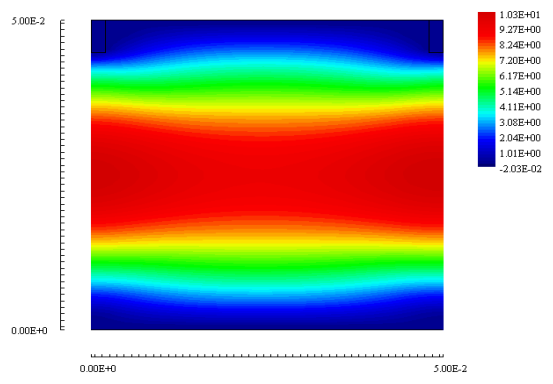

(a)

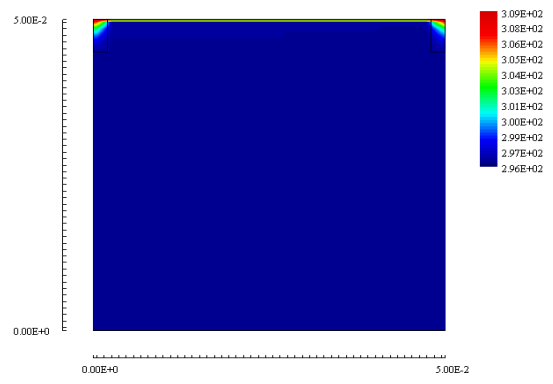

(b)

Figure 4: Two-dimensional distributions for (a) u-velocity and (b) temperature by the low Reynolds number $\mathrm{k}-\varepsilon$ model based on periodic flow condition.

\subsection{Standard $k-\varepsilon$ model}

Figure 5 shows the two-dimensional distribution for both velocity and temperature similar to Fig. 3 (a), but the thicknesses of the boundary layers for both velocity and temperature are smaller than the thicknesses of boundary layers in Fig. 3 (a). This can be explained due to the wall function approach in the standard k- $\varepsilon$ model instead of damping functions which are used in the low Re number k- $\varepsilon$ model.

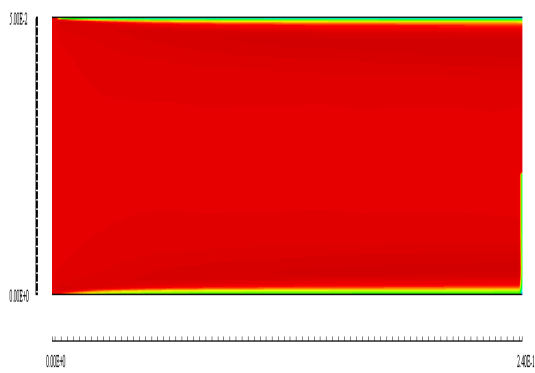

(a)

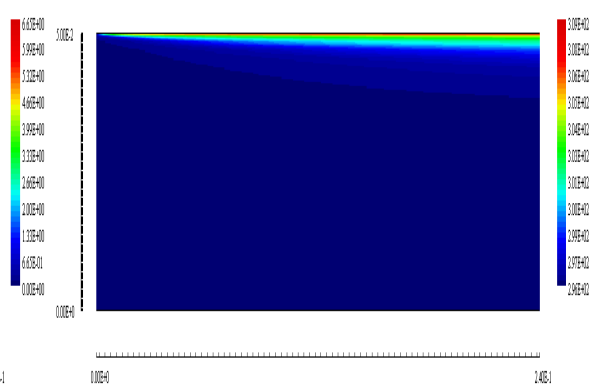

(b)

Figure 5: Two-dimensional distributions for (a) u-velocity and (b) temperature by the standard k- $\varepsilon$ model.

\section{Acknowledgement}

Financial support has been received by the Erasmus Mundus Windows Programme and the Swedish Energy Agency. 


\section{References}

[1] Acharya, S., Dutta, S., Myrum, T., \& Baker, R.S., Periodically developed flow and heat transfer in a ribbed duct, International Journal of Heat and Mass Transfer, 36, pp. 2069-2082, 1992.

[2] Liou T.M., Hwang J.J., \& Chen S.H., Simulation and measurement of enhanced turbulent heat transfer in a channel with periodic ribs on one principal wall, International Journal of Heat and Mass Transfer, 36(2), pp. 507-517, 1993.

[3] Prakash, C., \& Zerkle, R., Prediction of turbulent flow and heat transfer in a ribbed rectangular duct with and with- out rotation, ASME Journal of Turbomachinery, 117, pp. 25-264,1995.

[4] Ooi, A., Iaccarino, G., Durbin, P.A., \& Behnia, M., Reynolds averaged simulation of flow and heat transfer in ribbed ducts. International Journal of Heat and Fluid Flow, 23, pp. 750-757, 2002.

[5] Besserman, D.L., \& Tanrikut, S., Comparison of heat transfer measurements with computations for turbulent flow around a 180 degree bend, ASME Journal of Turbomachinery, 114,pp. 754-760,1992.

[6] Iacovides, H., The computation of turbulent flow through stationary and rotating u-bends with rib-roughened surfaces, International Journal for Numerical Methods in Fluids, 29, pp. 865-876, 1999.

[7] Sewall, E., \& Tafti, D., Large eddy simulation of flow and heat transfer in the 180-Deg bend region of a stationary gas turbine blade ribbed internal cooling duct, ASME Journal of Turbomachinery, 128, pp. 763-771. 2006

[8] Murata, A., \& Mochizuki, S., Large eddy simulation with a dynamic subgrid-scale model of turbulent heat transfer in an orthogonally rotating rectangular duct with transverse rib turbulators. International Journal of Heat and Mass Transfer, 43, pp. 1243-1259, 2000.

[9] Murata, A., \& Mochizuki, S., Effect of cross-sectional aspect ratio on turbulent heat transfer in an orthogonally rotating rectangular duct with angled rib turbulators, International Journal of Heat and Mass Transfer, 46, pp. 3119--3133, 2003.

[10] Versteeg, H.K., \& Malalasekera, W., An Introduction to Computational fluid Dynamic The Finite Volume Method, 2nd Edition, Pearson Education Limited: England, 2007. 